\title{
Comprehensive Analysis of the Clinical Significance and Prognostic Value of the CBX Family in Cervical Squamous Cell Carcinoma and Endocervical Adenocarcinoma (CESC)
}

\section{Pei Zhou}

First Affiliated Hospital of Anhui Medical University

Cong Ma ( $\sim$ congm1995@163.com )

First Affiliated Hospital of Anhui Medical University https://orcid.org/0000-0002-7912-1424

\section{Caiyun Wu}

First Affiliated Hospital of Anhui Medical University

Jing Yuan

First Affiliated Hospital of Anhui Medical University

\section{Research}

Keywords: CESC, chromobox (CBX), prognosis, biomarkers

Posted Date: April 14th, 2021

DOI: https://doi.org/10.21203/rs.3.rs-406048/v1

License: (c) (i) This work is licensed under a Creative Commons Attribution 4.0 International License.

Read Full License 


\section{Abstract}

Background: Cervical squamous cell carcinoma and endocervical adenocarcinoma (CESC) is the second most common type of cancer among gynecologic malignancies worldwide. Chromobox (CBX) family proteins are associated with the regulation of tumorigenesis, metastasis, and evolution of various cancers.

Methods: The clinical features, expression levels, and prognostic value of CBXs in CESC were analyzed through several databases, including ONCOMINE, GEPIA, HPA, UALCAN, cBioPortal, Kaplan-Meier plotter, and Metascape.

Results: We concluded that the expression level of $\mathrm{CBX} 2 / 4 / 8$ was upregulated, while the expression level of CBX6/7 was downregulated in CESC specimens. Immune infiltration analysis revealed that CBX1/2/3/4/5/6/8 proteins were downregulated in normal cervical tissues, and upregulated in CESC specimens. In contrast, CBX7 protein expression was significantly higher in normal adjacent cervical tissues and was not detected in CESC tissues. CBX1/3/6 mRNA expression was significantly correlated with the pathological stage of CESC. Prognostic analysis showed that patients with high CBX7 levels of CESC had a favorable prognosis.

Conclusions: Our study indicated that CBX7 could be an attractive biomarker for the prognosis of CESC.

\section{Introduction}

Cervical squamous cell carcinoma and endocervical adenocarcinoma (CESC) is the second most common type of cancer among gynecologic malignancies worldwide [1, 2]. The estimated annual agestandardized incidence rate is $15 / 100000$ women globally, and CESC predominantly occurs in women aged 20-39 years. In recent years, due to advances in diagnosis and treatments, CESC survival rates have improved[3, 4].

Although it has been discovered that CESC is caused by HPV (human papillomavirus) infection, there is a lack of effective biomarkers to detect CESC patients early $[5,6]$. Patients with CESC have a poor prognosis once they have metastatic tumors or are in advanced stages. On account of tumor heterogeneity, there is a critical need for valid biomarkers to improve individual treatment and the prediction of outcomes.

Additionally, abnormal epigenetic regulation plays a prominent role in tumorigenesis [7]. Chromobox (CBX) family proteins are an important component of the polycomb group (PcG) as an epigenetic regulatory complex that inhibits the transcription of target genes by modifying chromatin. Recently, eight members of the CBXs family of proteins have been identified that contain a similar single $\mathrm{N}$-terminal chromosomal domain. All of them participate in gene expression, regulation of heterochromatin, and developmental programs [8-10]. In previous studies, members of the CBXs family were found to have 
prognostic value. For example, $\mathrm{CBX} 1$ protein expression is increased in human hepatocellular carcinoma tissues, CBX2 is significantly elevated in human prostate cancer specimens $[11,12]$.

Nevertheless, the roles of CBXs family proteins in the carcinogenesis and progression of CESC have not been completely investigated. Therefore, in our research, we extended the research field to CESC based on various online databases to determine the prognostic values of distinct CBX family members.

\section{Materials And Methods}

\section{ONCOMINE database}

The ONCOMINE database (www.oncomine.org) is a web-based DNA and RNA sequence analysis data mining platform and cancer gene chip database that facilitates genome-wide expression analysis [13]. In our study, the transcriptional levels of CBXs between cancer tissues and their corresponding adjacent normal samples were analyzed using the ONCOMINE database. Student's t-tests were used to compare differences in transcription levels ( $p$-value: 0.01 , fold change: 1.5 , gene rank: top $10 \%$, data type: mRNA).

\section{UALCAN}

UALCAN (http://ualcan.path.uab.edu) is an interactive online tool. It can be used to analyze relative gene expression in matched samples and estimate associations between transcriptional expression and clinicopathological parameters of patient survival time [14]. In this study, UALCAN was used to analyze the relationship between the mRNA expression level of eight CBX family members and the grade of CESC cancer. The student's t-test was used to compare the differences, and a p-value $<0.01$ was considered statistically significant.

\section{Human Protein Atlas (HPA)}

The Human Protein Atlas (https://www.proteinatlas.org) is a web portal that contains nearly 20 types of cancer and transcriptome analysis of 8000 patient data and is based on immunohistochemical expression [15]. Users can identify the protein expression patterns of specific genes in specific types of tumors. In this study, we obtained immunohistochemical images to directly compare the expression of different CBX family members in normal tissues with CESC specimens.

\section{GEPIA database}

The GEPIA (Gene Expression Profiling Interactive Analysis) database (http://gepia.cancer-pku.cn/) is an interactive online tool containing RNA expression data and provides fast and customizable capabilities based on Cancer Genome Atlas (TCGA) and Genotypic Tissue Expression (GTEX) data [16]. Using this database, we successively analyzed the differences in expression between CESC and normal tissues in eight members of the CBX family. Expression fold changes $\geq 1.5$ and $p$-value $<0.05$ were considered statistically significant. 


\section{Kaplan-Meier plotter}

Kaplan-Meier plotter (http://kmplot.com/analysis/) is an online tool to evaluate the influence of 54,000 genes on 21 types of cancer survival and draw survival plots with gene expression data and survival information $[17,18]$. The results are shown by plotting a survival curve and simultaneously analyzing hazard ratios (HRs) with 95\% confidence intervals (Cls) and log-rank p-values. When a p-value $<0.05$, a statistically significant difference was considered.

\section{cBioPortal}

cBioPortal (www.cbioportal.org) is an online open-access portal that provides a way to visualize, explore, and analyze multidimensional cancer genomics data [19]. In our study, we compared the frequency of mutations in the CBXS gene in CESC and summarized all associated genomic changes.

\section{Metascape}

Metascape (http://metascape.org) was used for functional enrichment and pathway annotation of genes [20]. We researched the genes in the resulting CBX family coexpression network in STRING employing the Gene Ontology (GO) and Kyoto Encyclopedia of Genes and Genomes (KEGG) pathways.

\section{Results}

\section{The expression levels of eight CBX family members in patients with CESC}

ONCOMINE, GEPIA, and HPA were used to analyze the expression levels of the CBX family in CESC. In the ONCOMINE database, the mRNA expression of eight CBX family members in various tumors was analyzed and compared with normal tissues (Fig. 1 and Table 1). Concerning CBX1, we found overexpression levels in the Dohun cervical statistics dataset $(F C=3.762, p=1.72 E-08)$. Regarding $C B X 2$, low expression levels were found in the Luigi cervical statistics dataset $(F C=-1.794, p=0.000145)$. Notably, we found that CBX3/5 mRNA is highly expressed in CESC tissues in multiple datasets. In the Yali dataset, for $\mathrm{CBX} 3$, compared to normal tissue, cervical squamous cell carcinoma (CSCC) tissues and high-grade cervical squamous intraepithelial neoplasias (HGCEIN) had fold changes of 1.911 ( $p=1.26 \mathrm{E}-$ 08 ) and $1.576(p=0.003)$, respectively. While Dohun found that CBX3 mRNA expression had a 2.701-fold increase in cervical cancer samples $(p=2.37 \mathrm{E}-09)$, Luigi and Petra found a 1.812-fold and 2.202-fold increase in CBX1 mRNA expression in CESC tissues, respectively $(p=0.00000164 / p=0.0000113)$. Similarly, Yali observed a 2.166-fold/2.18-fold increase in CBX5 mRNA expression in HGCEIN/CSCC samples $(p=0.000528 / p=0.000545)$, while Dohun observed a 2.78-fold increase in CBX5 mRNA expression in cervical cancer samples $(p=1.29 \mathrm{E}-08)$ and Luigi observed a 2.094-fold increase in CBX5 mRNA expression in CSCC samples $(p=0.00000415)$. Importantly, Petra observed a -2.132 -fold increase in CBX7 mRNA expression in CSCC samples $(p=1.03 E-12)$ [22-25]. 
Table 1

Effective variations in CBX expression at the transcription level between CESC and normal cervical tissues (ONCOMINE database).

\begin{tabular}{|lllll|}
\hline CBXs & Types of CESC & Fold Change & $p$-value & Ref (PMID:) \\
\hline CBX1 & Cervical Cancer & 3.726 & $1.72 \mathrm{E}-08$ & 17510386 \\
\hline CBX2 & CSCC & -1.794 & 0.000145 & 18506748 \\
\hline CBX3 & CSCC & 1.911 & $1.26 \mathrm{E}-08$ & 17974957 \\
& HGCSIN & 1.576 & 0.003 & 17974957 \\
& Cervical Cancer & 2.701 & $2.37 \mathrm{E}-09$ & 17510386 \\
& CSCC & 1.812 & 0.00000164 & 18506748 \\
\hline CBX5 & CSCC & 2.202 & 0.0000113 & 18191186 \\
\hline & HGCSIN & 2.166 & 0.000528 & 17974957 \\
\hline & CSCC & 2.18 & 0.000545 & 17974957 \\
\hline $\begin{array}{l}\text { CBX7 } \\
\text { CESC: Cervical Cancer }\end{array}$ & 2.78 & $1.29 \mathrm{E}-08$ & 17510386 \\
\hline $\begin{array}{l}\text { Cervical Squamous Intraepithelial Neoplasia; CSCC: Cervical Squamous Cell Carcinoma; CBX: } \\
\text { chromobox }\end{array}$ & 2.094 & 0.00000415 & 18506748 \\
\hline
\end{tabular}

As shown in Fig. 2, compared to adjusted normal tissues, the GEPIA database indicated significant mRNA overexpression of CBX2/4/8 (Fig. 2B, 2D, 2H) in CESC samples $(p<0.05)$. However, the expression levels of CBX6 (Fig. 2F) and CBX7 (Fig. 2G) were reduced in CESC tissues $(p<0.05)$.

After inspecting the mRNA expression patterns of CBXs in CESC, we subsequently attempted to analyze the expression pattern of CBXs family proteins in CESC by HPA (Human Protein Atlas). As shown in Fig. 3, the normal cervical tissues presented medium protein expression of $C B X 1 / 2 / 3 / 4 / 5$, while medium and high protein expression was discovered in CESC tissues (Fig. 3A-3E). Analogously, low protein expression of $\mathrm{CBX} 6 / 8$ was observed in normal cervical tissues, while medium and high protein expression of CBX6/8 was observed in CESC samples (Fig. 3F, 3H). In contrast, we found that the CBX7 protein was medially expressed in healthy cervical tissues and was not detected in CESC tissues (Fig. 3G).

The clinicopathological parameters associated with CESC patients were investigated using models of mRNA expression of eight members of the CBX family 
Afterward, we analyzed the correlation between CBXs family mRNA expression levels and clinicopathological parameters in patients with CESC by GEPIA and UALCAN, containing patients' cancer stages and tumor grades. In the Violin plot (Fig. 4), we found that the expression of CBX2/6/8 mRNA was correlated with the pathological stage of CESC patients.

Figure 5 shows the relationship of the mRNA expression level of distinct CBX family members with the tumor grades of CESC patients. The mRNA expression levels of CBX1/3/6 were related to tumor grade. When the tumor grade advanced, $\mathrm{CBX} 1 / 3$ tended to have a higher mRNA expression level, and CBX6 had the opposite trend. In tumor grade 4, we found the highest mRNA expression of CBX1/2/3 (Fig. 5A, 5B, $5 C$ ). The highest mRNA expression of $C B X 4 / 5 / 8$ was found in tumor grade 2 (Fig. 5D, 5E, 5H), while the highest mRNA expression of CBX6/7 was found in normal tissues (Fig. 5F, 5G).

In summary, the results showed that the mRNA expression of several CBX family members was significantly correlated with clinicopathological parameters in patients with CESC.

\section{The relationship between CBXs mRNA expression levels and prognosis in patients with CESC}

Next, we analyzed the relationship between the CBXs mRNA expression levels and the prognosis of patients with CESC (Fig. 6). Distinctly, CBX1/2/3/4/5/6/8 mRNA expression showed no correlation with prognosis in CESC patients (Fig. 6A-6G), while higher mRNA expression of CBX7 ( $\mathrm{HR}=0.43,95 \% \mathrm{Cl}$ : $0.26-0.69, p=0.00042$ ) was significantly correlated with favorable OS (overall survival) in CESC patients (Fig. $6 \mathrm{H}$ ). The results showed that the mRNA expression level of CBX7 could be used as an important biomarker to predict the OS of patients with CESC.

\section{Cbxs Family Gene Mutations In The Patients With Cesc}

CBXs family gene mutations in the patients with CESC We analyzed genetic changes in the patients using the cBioPortal tool and found mutations in the CBXS gene in 64 of 188 patients, with a mutation rate of $34 \%$. In addition, the mutation rates of the CBX4, CBX1, CBX2, and CBX3 genes were $11 \%, 9 \%, 7 \%$, and $7 \%$, respectively (Fig. 7 ).

\section{Predicted Functions And Pathways Of The Mutations In Cbxs}

After analyzing CBXs family gene mutations in patients with CESC, we analyzed neighboring genes associated with mutations in the CBXs family of genes and constructed a complete network. As shown in Fig. $8 \mathrm{~A}$, all of the genes encode proteins that belong to the polycomb group $(\mathrm{PcG})$ and form multiprotein complexes. They are important for the transcriptional repression of various genes involved in development and cell proliferation. Moreover, the functions of CBXs and their altered neighboring genes were analyzed by GO and KEGG in Metascape. In Fig. 8B, biological processes such as 
GO:0035102(PRC1 complex), G0:0016574(histone ubiquitination), G0:0070317(negative regulation of G0-G1 transition), G0:0000792(heterochromatin), G0:0042393(histone binding), and GO:0003714(transcription corepressor activity) are shown. Additionally, ko04550: signaling pathways regulating the pluripotency of stem cells came up in the KEGG analysis.

\section{Discussion}

CESC is one of the deadliest diseases originating from cervical cells. Squamous cell carcinoma and are the most common pathological subtypes accounting for approximately $70 \%$, and adenocarcinoma accounting for approximately $25 \%$ of all CESC. Since the 3-year to 5-year survival rate of patients with CESC in many developing countries is $<50 \%$, early detection, effective therapy, and precise prediction of the outcome are of great significance for CESC patients $[1,5,21]$.

Although most cervical cancer cases are due to human papillomavirus (HPV) infection, additional genetic and epigenetic changes are required for cervical carcinogenesis [22]. Recently, advances in understanding the biology of CESC have shown that epigenetic alterations are common in the tumorigenesis of CESC and metastasis [23]. Therefore, novel biomarkers related to the prognosis and treatment of CESC are urgently needed.

In our study, the expression level, mutation status, and prognostic value of eight members of the CBX family in CESC were analyzed. We mainly focused on the relationship between the CBXs mRNA expression level and the prognosis of patients with CESC. We revealed that CESC patients with high CBX7 levels had a favorable prognosis, demonstrating CBX7 as a potential prognostic biomarker for CESC.

The CBX family is a canonical component of PcG and is implicated in the development of diversified cancers [24]. CBX1 is a member of the heterochromatin protein 1 (HP1) family, also known as HP1 3 . Yang et al. found that the expression level of CBX1 was significantly increased in HCC tissues and cell lines and was significantly correlated with tumor size, degree of differentiation, and tumor aggressiveness [11]. Analogously, Shiota et al. found that CBX1 expression was increased in prostate cancer samples. Additionally, its high expression could enhance the activity of the androgen receptor pathway and accelerate the growth of prostate cancer [25].

Recently, Clermont et al. conducted a Geno transcriptomic meta-analysis and found high CBX2 mRNA expression in human cancer. In particular, overexpression and amplification of CBX2 were significantly correlated with metastatic progression and a shorter OS of BC patients [26]. Meanwhile, Wheeler et al. found that CBX2 upregulation promotes high-grade serous ovarian carcinoma by inducing a stem-like transcriptional profile and inhibiting anoikis [27]. Mechanistically, studies from Zhao et al., Ma et al., and Chen et al. showed that CBX3 is overexpressed in gliomas, osteosarcoma, and pancreatic adenocarcinoma, and upregulated CBX3 is indicative of a worse OS[28-30].

CBX4 has been thoroughly studied in a variety of cancers. Jiao et al. and Wang et al. respectively found that CBX4 was highly expressed in clinical specimens and multiple hepatocellular carcinoma cells. It was 
also associated with clinical parameters, including the a-fetoprotein level, tumor size, lymph node metastasis, distant metastasis, pathologic differentiation, shorter OS, and relapse-free survival (RFS) [31, 32]. Additional studies carried out by Li et al. showed that CBX4 was overexpressed in HCC mice and promoted tumorigenesis by increasing VEGF production and angiogenesis, while knockdown of CBX4 suppressed the tumorigenicity of CBX4 [33]. Furthermore, Hu et al. indicated that CBX4 is overexpressed in lung cancer cells and promotes proliferation and metastasis by regulating the BMI-1 [34]. CBX5 also known as heterochromatic protein $1 \mathrm{a}(\mathrm{HP} 1 \mathrm{a})$, and overexpression of CBX5 was found in many types of malignant tumors, including pancreatic cancers, breast cancers, and lung cancers [35].

In a previous report, Zheng et al. found that the higher expression of CBX6 in HCC promotes the growth of hepatocellular carcinoma cells by regulating the pathway: S100A9/NF-KB/MAPK and hepatocellular carcinoma patients with higher CBX6 expression had significantly shorter RFS and OS [36]. Studies carried out by $\mathrm{Li}$ et al. showed that CBX6 was downregulated in glioblastoma multiforme cells and clinical tissues. In addition, overexpression of CBX6 suppressed the proliferation of glioblastoma multiforme cells [37].

Different characteristics of CBX7 have been found in multiple types of human cancers. Pallante et al. reported that $\mathrm{CBX7}$ is decreased in diversified human malignant tumors, including thyroid cancers, breast cancers, lung carcinomas, colorectal cancers, and bladder cancers. Additionally, decreased expression of CBX7 has been shown to significantly correlate with higher metastasis and a worse prognosis of patients. Mechanistically, CBX7 inhibits the expression of proliferation and migration genes containing CCNE and SPP1 by counteracting the carcinogenesis of HMGA proteins [38]. Shinjo et al. found that higher expression of $\mathrm{CBX} 7$ is significantly associated with worse overall and progression-free survival rates in ovarian cancer patients [39]. And Li et al. found that CBX7 inhibits tumor growth and metastatic potential by binding to the E-box to inhibit TWIST-1 function in ovarian cancer [40]. In our study, we found that CBX7 expression was decreased in CESC tissues compared to adjusted normal tissues, while higher mRNA expression of CBX7 was significantly associated with a favorable OS (overall survival) in CESC patients. In addition, The CBX7 protein expression was not detected in CESC tissues demonstrated by immunohistochemistry pictures. These results indicate that $\mathrm{CBX7}$ is an effective marker for predicting the outcomes of patients.

Similarly, CBX8 plays a significant role in lymphoma, breast carcinoma, esophageal squamous cell carcinoma, esophageal cancer, and other human cancers [41]. Studies carried out by Yuan et al. showed that upregulated CBX8 was associated with the progression of the stages in muscle-invasive bladder cancer tissues by repressing the p53 pathway [42]. Coincidentally, CBX8 inhibits metastasis by suppressing the Snail pathway in esophageal squamous cell carcinoma while promoting cell proliferation [43].

In conclusion, we found that high expression of CBX7 mRNA is significantly related to the favorable prognosis and survival of CESC patients. Other members of the CBX family have no obvious relationship with prognosis and survival. Certainly, there are some limitations to our study, and all of the data 
researched in our study were acquired from online databases. Further research requires a larger sample size to test our findings and to explore the clinical application and potential mechanisms of CBX members in the treatment of CESC.

\section{Abbreviations}

CESC:cervical squamous cell carcinoma and endocervical adenocarcinoma;CBX: chromobox; HPV:human papillomavirus; HPA: The Human Protein Atlas; GEPIA: Gene Expression Profiling Interactive Analysis; GO: Gene Ontology; KEGG: Kyoto Encyclopedia of Genes and Genomes; OS: Overall survival; RFS:relapse-free survival.

\section{Declarations}

\section{Authors' contributions}

$\mathrm{PZ}$ and $\mathrm{CM}$ are involved in designing and writing articles, $\mathrm{CM}$ and $\mathrm{CYW}$ were

involved in the data curation, formal analysis, and methodologyand. JY designed and reviewed the manuscript. The authors read and approved the final manuscript.

\section{Funding}

This work was supported by the Non-profit Central Research Institute Fund of Chinese Academy of Medical Sciences(2019PT310002), and Research Fund of Anhui Institute of translational medicine (ZHYX2020A001).

\section{Availability of data and materials}

All the data in this research are available from ONCOMINE, GEPIA, HPA, UALCAN, cBioPortal, Kaplan-Meier plotter, and Metascape database

\section{Ethics approval and consent to participate}

Not applicable.

\section{Consent for publication}

Not applicable.

\section{Competing interests}

The authors declare that they have no competing interests.

\section{References}


1. Small W Jr, Bacon MA, Bajaj A, Chuang LT, Fisher BJ, Harkenrider MM, et al: Cervical cancer: A global health crisis. Cancer 2017, 123:2404-2412. doi:10.1002/cncr.30667.

2. Koh WJ, Abu-Rustum NR, Bean S, Bradley K, Campos SM, Cho KR, et al. Cervical Cancer, Version 3.2019, NCCN Clinical Practice Guidelines in Oncology. J Natl Compr Canc Netw. 2019;17:64-84. doi:10.6004/jnccn.2019.0001.

3. Siegel RL, Miller KD, Jemal A. Cancer statistics, 2020. CA: A Cancer Journal for Clinicians 2020, 70:7-30. doi:10.3322/caac.21590.

4. Arbyn M, Weiderpass E, Bruni L, de Sanjosé S, Saraiya M, Ferlay J, et al. Estimates of incidence and mortality of cervical cancer in 2018: a worldwide analysis. The Lancet Global Health. 2020;8:e191203. doi:10.1016/s2214-109x(19)30482-6.

5. Cohen PA, Jhingran A, Oaknin A, Denny L. Cervical cancer. The Lancet 2019, 393:169-182. doi:10.1016/s0140-6736(18)32470-x.

6. Sawaya GF, Smith-McCune K, Kuppermann M. Cervical Cancer Screening: More Choices in 2019. Jama 2019, 321:2018-2019. doi:10.1001/jama.2019.4595.

7. Dawson MA, Kouzarides T. Cancer epigenetics: from mechanism to therapy. Cell 2012, 150:12-27. doi:10.1016/j.cell.2012.06.013.

8. Kim J, Kingston RE. The CBX family of proteins in transcriptional repression and memory. Journal of Biosciences 2020, 45. doi:10.1007/s12038-019-9972-5.

9. Wotton D, Merrill JC. Pc2 and SUMOylation. Biochem Soc Trans. 2007;35:1401-4. doi:10.1042/bst0351401.

10. Vandamme J, Volkel P, Rosnoblet C, Le Faou P, Angrand PO. Interaction proteomics analysis of polycomb proteins defines distinct PRC1 complexes in mammalian cells. Mol Cell Proteomics. 2011;10::M110 002642. doi:10.1074/mcp.M110.002642.

11. Yang YF, Pan YH, Tian QH, Wu DC, Su SG. CBX1 Indicates Poor Outcomes and Exerts Oncogenic Activity in Hepatocellular Carcinoma. Transl Oncol 2018, 11:1110-1118. doi:10.1016/j.tranon.2018.07.002.

12. Clermont PL, Crea F, Chiang YT, Lin D, Zhang A, Wang JZ, et al. Identification of the epigenetic reader CBX2 as a potential drug target in advanced prostate cancer. Clin Epigenetics. 2016;8::16. doi:10.1186/s13148-016-0182-9.

13. Rhodes DR, Yu J, Shanker K, Deshpande N, Varambally R, Ghosh D, et al. ONCOMINE: a cancer microarray database and integrated data-mining platform. Neoplasia. 2004;6:1-6. doi:10.1016/s1476-5586(04)80047-2.

14. Chandrashekar DS, Bashel B, Balasubramanya SAH, Creighton CJ, Ponce-Rodriguez I, Chakravarthi B, et al: UALCAN: A Portal for Facilitating Tumor Subgroup Gene Expression and Survival Analyses. Neoplasia 2017, 19:649-658. doi:10.1016/j.neo.2017.05.002.

15. Asplund A, Edqvist PH, Schwenk JM, Ponten F. Antibodies for profiling the human proteome-The Human Protein Atlas as a resource for cancer research. Proteomics 2012, 12:2067-2077. doi:10.1002/pmic.201100504. 
16. Tang Z, Li C, Kang B, Gao G, Li C, Zhang Z. GEPIA: a web server for cancer and normal gene expression profiling and interactive analyses. Nucleic Acids Res. 2017;45::W98-102. doi:10.1093/nar/gkx247.

17. Gyorffy B, Lanczky A, Eklund AC, Denkert C, Budczies J, Li Q, et al. An online survival analysis tool to rapidly assess the effect of 22,277 genes on breast cancer prognosis using microarray data of 1,809 patients. Breast Cancer Res Treat. 2010;123:725-31. doi:10.1007/s10549-009-0674-9.

18. Szász AM, Lánczky A, Nagy Á, Förster S, Hark K, Green JE, et al: Cross-validation of survival associated biomarkers in gastric cancer using transcriptomic data of 1,065 patients. Oncotarget 2016, 7:49322-49333. doi:10.18632/oncotarget.10337.

19. Gao J, Aksoy BA, Dogrusoz U, Dresdner G, Gross B, Sumer SO, et al. Integrative analysis of complex cancer genomics and clinical profiles using the cBioPortal. Sci Signal. 2013;6:pl1.

doi:10.1126/scisignal.2004088.

20. Zhou Y, Zhou B, Pache L, Chang M, Khodabakhshi AH, Tanaseichuk O, et al. Metascape provides a biologist-oriented resource for the analysis of systems-level datasets. Nat Commun. 2019;10::1523. doi:10.1038/s41467-019-09234-6.

21. Tsikouras P, Zervoudis S, Manav B, Tomara E, latrakis G, Romanidis C, et al. Cervical cancer: screening, diagnosis and staging. J buon. 2016;21:320-5.

22. Olusola P, Banerjee HN, Philley JV, Dasgupta S. Human Papilloma Virus-Associated Cervical Cancer Health Disparities Cells 2019, 8. doi:10.3390/cells8060622.

23. Fang J, Zhang H, Jin S. Epigenetics and cervical cancer: from pathogenesis to therapy. Tumour Biol. 2014;35:5083-93. doi:10.1007/s13277-014-1737-z.

24. Ma RG, Zhang Y, Sun TT, Cheng B. Epigenetic regulation by polycomb group complexes: focus on roles of CBX proteins. J Zhejiang Univ Sci B. 2014;15:412-28. doi:10.1631/jzus.B1400077.

25. Shiota M, Song Y, Yokomizo A, Tada Y, Kuroiwa K, Eto M, et al. Human heterochromatin protein 1 isoform HP1beta enhances androgen receptor activity and is implicated in prostate cancer growth. Endocr Relat Cancer. 2010;17:455-67. doi:10.1677/ERC-09-0321.

26. Clermont PL, Sun L, Crea F, Thu KL, Zhang A, Parolia A, et al. Genotranscriptomic meta-analysis of the Polycomb gene CBX2 in human cancers: initial evidence of an oncogenic role. Br J Cancer. 2014;111:1663-72. doi:10.1038/bjc.2014.474.

27. Wheeler LJ, Watson ZL, Qamar L, Yamamoto TM, Post MD, Berning AA, et al: CBX2 identified as driver of anoikis escape and dissemination in high grade serous ovarian cancer. Oncogenesis 2018, 7:92. doi:10.1038/s41389-018-0103-1.

28. Zhao SP, Wang F, Yang M, Wang XY, Jin CL, Ji QK, et al. CBX3 promotes glioma U87 cell proliferation and predicts an unfavorable prognosis. J Neurooncol. 2019;145:35-48. doi:10.1007/s11060-01903286-W.

29. Ma C, Nie XG, Wang YL, Liu XH, Liang X, Zhou QL, et al. CBX3 predicts an unfavorable prognosis and promotes tumorigenesis in osteosarcoma. Mol Med Rep. 2019;19:4205-12. doi:10.3892/mmr.2019.10104. 
30. Chen LY, Cheng CS, Qu C, Wang P, Chen H, Meng ZQ, et al: Overexpression of CBX3 in Pancreatic Adenocarcinoma Promotes Cell Cycle Transition-Associated Tumor Progression. Int J Mol Sci 2018, 19. doi:10.3390/ijms19061768.

31. Wang B, Tang J, Liao D, Wang G, Zhang M, Sang Y, et al. Chromobox homolog 4 is correlated with prognosis and tumor cell growth in hepatocellular carcinoma. Ann Surg Oncol. 2013;20(Suppl 3):684-92. doi:10.1245/s10434-013-3171-7.

32. Jiao HK, Xu Y, Li J, Wang W, Mei Z, Long XD, et al. Prognostic significance of Cbx4 expression and its beneficial effect for transarterial chemoembolization in hepatocellular carcinoma. Cell Death Dis. 2015;6:e1689. doi:10.1038/cddis.2015.57.

33. Li J, Xu Y, Long XD, Wang W, Jiao HK, Mei Z, et al: Cbx4 governs HIF-1alpha to potentiate angiogenesis of hepatocellular carcinoma by its SUMO E3 ligase activity. Cancer Cell 2014, 25:118131. doi:10.1016/j.ccr.2013.12.008.

34. Hu C, Zhang Q, Tang Q, Zhou H, Liu W, Huang J, et al. CBX4 promotes the proliferation and metastasis via regulating BMI-1 in lung cancer. J Cell Mol Med. 2020;24:618-31. doi:10.1111/jcmm.14771.

35. Vad-Nielsen J, Nielsen AL. Beyond the histone tale: HP1alpha deregulation in breast cancer epigenetics. Cancer Biol Ther. 2015;16:189-200. doi:10.1080/15384047.2014.1001277.

36. Zheng H, Jiang WH, Tian T, Tan HS, Chen Y, Qiao GL, et al: CBX6 overexpression contributes to tumor progression and is predictive of a poor prognosis in hepatocellular carcinoma. Oncotarget 2017, 8:18872-18884. doi:10.18632/oncotarget.14770.

37. Li G, Warden C, Zou Z, Neman J, Krueger JS, Jain A, et al. Altered expression of polycomb group genes in glioblastoma multiforme. PLoS One. 2013;8:e80970. doi:10.1371/journal.pone.0080970.

38. Pallante P, Forzati F, Federico A, Arra C, Fusco A. Polycomb protein family member CBX7 plays a critical role in cancer progression. Am J Cancer Res. 2015;5:1594-601.

39. Forzati F, Federico A, Pallante P, Fedele M, Fusco A. Tumor suppressor activity of CBX7 in lung carcinogenesis. Cell Cycle 2012, 11:1888-1891. doi:10.4161/cc.20022.

40. Li J, Alvero AB, Nuti S, Tedja R, Roberts CM, Pitruzzello M, et al: CBX7 binds the E-box to inhibit TWIST-1 function and inhibit tumorigenicity and metastatic potential. Oncogene 2020, 39:39653979. doi:10.1038/s41388-020-1269-5.

41. Lin K, Zhu J, Hu C, Bu F, Luo C, Zhu X, et al. Comprehensive analysis of the prognosis for chromobox family in gastric cancer. J Gastrointest Oncol. 2020;11:932-51. doi:10.21037/jgo-20-208.

42. Yuan GJ, Chen X, Lu J, Feng ZH, Chen SL, Chen RX, et al. Chromobox homolog 8 is a predictor of muscle invasive bladder cancer and promotes cell proliferation by repressing the $p 53$ pathway. Cancer Sci. 2017;108:2166-75. doi:10.1111/cas.13383.

43. Wang G, Tang J, Zhan W, Zhang R, Zhang M, Liao D, et al. CBX8 Suppresses Tumor Metastasis via Repressing Snail in Esophageal. Squamous Cell Carcinoma Theranostics. 2017;7:3478-88. doi:10.7150/thno.20717. 


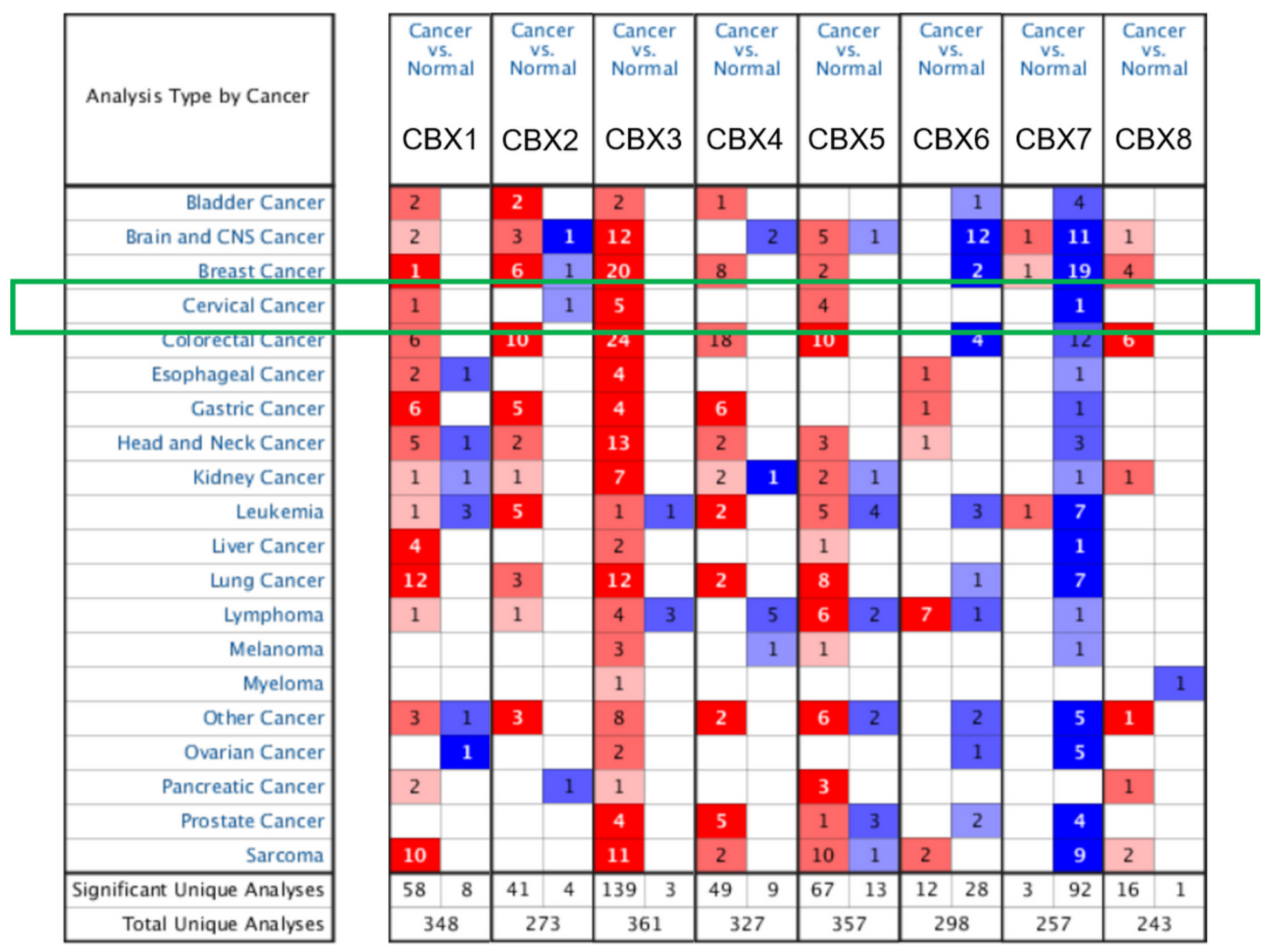

\section{Figure 1}

Transcriptional expression of CBXs in 20 different types of cancer diseases (ONCOMINE database). The difference in transcriptional expression was compared with Student's t-test. The cutoff p-value and fold change were as follows: p-value: 0.01 , fold change: 1.5 , gene rank: $10 \%$, data type: mRNA. 

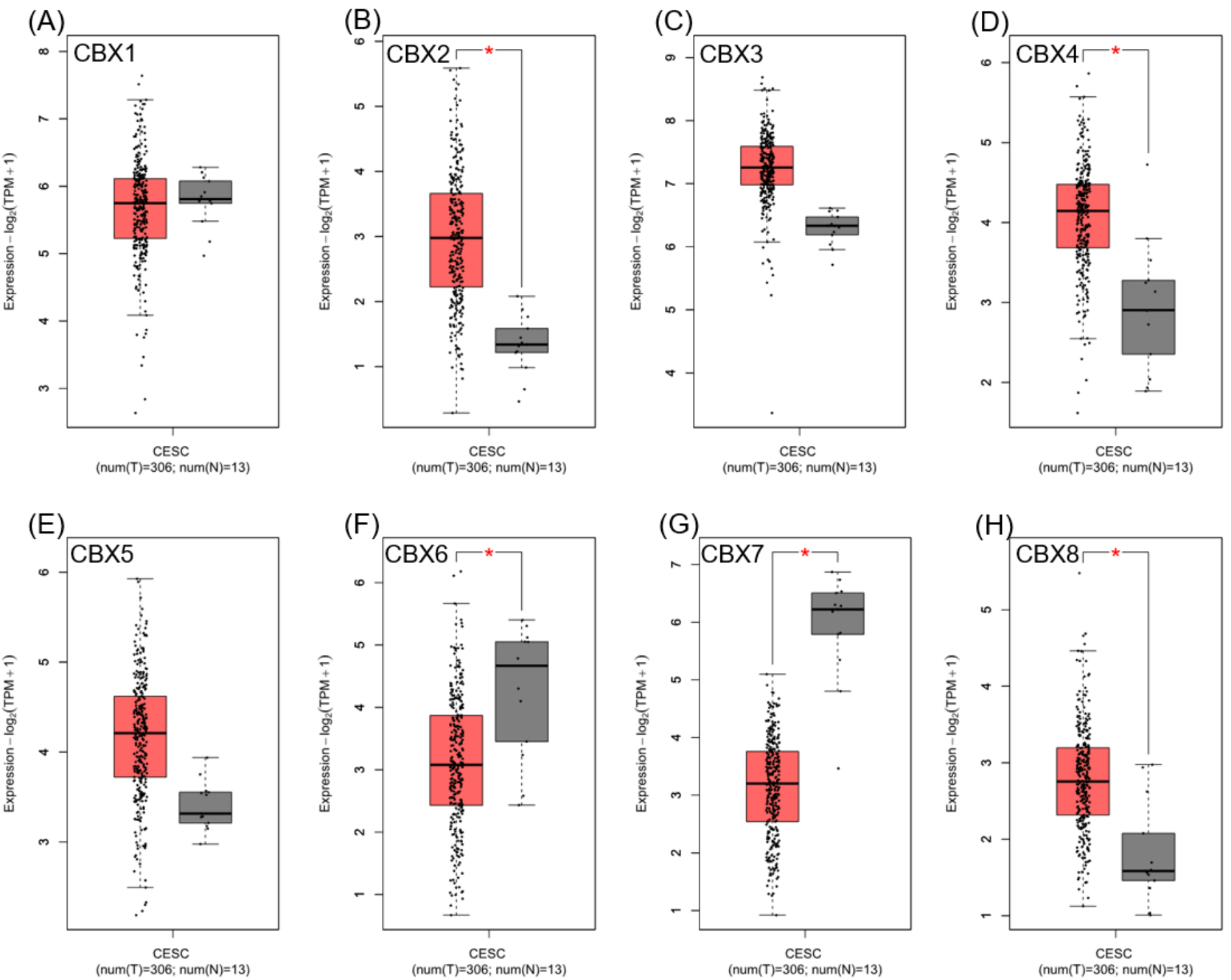

Figure 2

The expression of the CBX family in CESC (GEPIA). The plots derived from gene expression data for GEPIA comparing the expression of CBXs family in CESC with the p-value $<0.05$. *Indicates that the results are statistically significant.
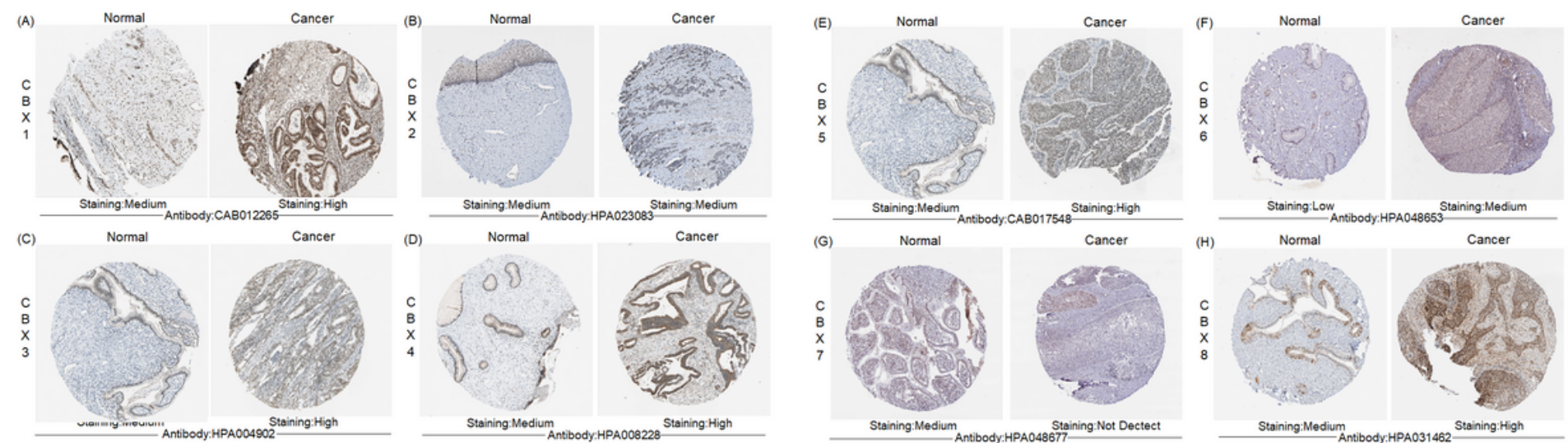
Figure 3

Immunohistochemistry images of distinct CBX family members in CESC tissues and normal cervical tissues by Human Protein Atlas (HPA). CBX1/3/4/5/6/8 protein expression was higher in CESC tissues $(A, C-F, H)$, and $C B X 2$ was not differentially expressed between CESC tissues and healthy cervical tissues (B). CBX7 protein expression was not significantly expressed in CESC tissues (G).
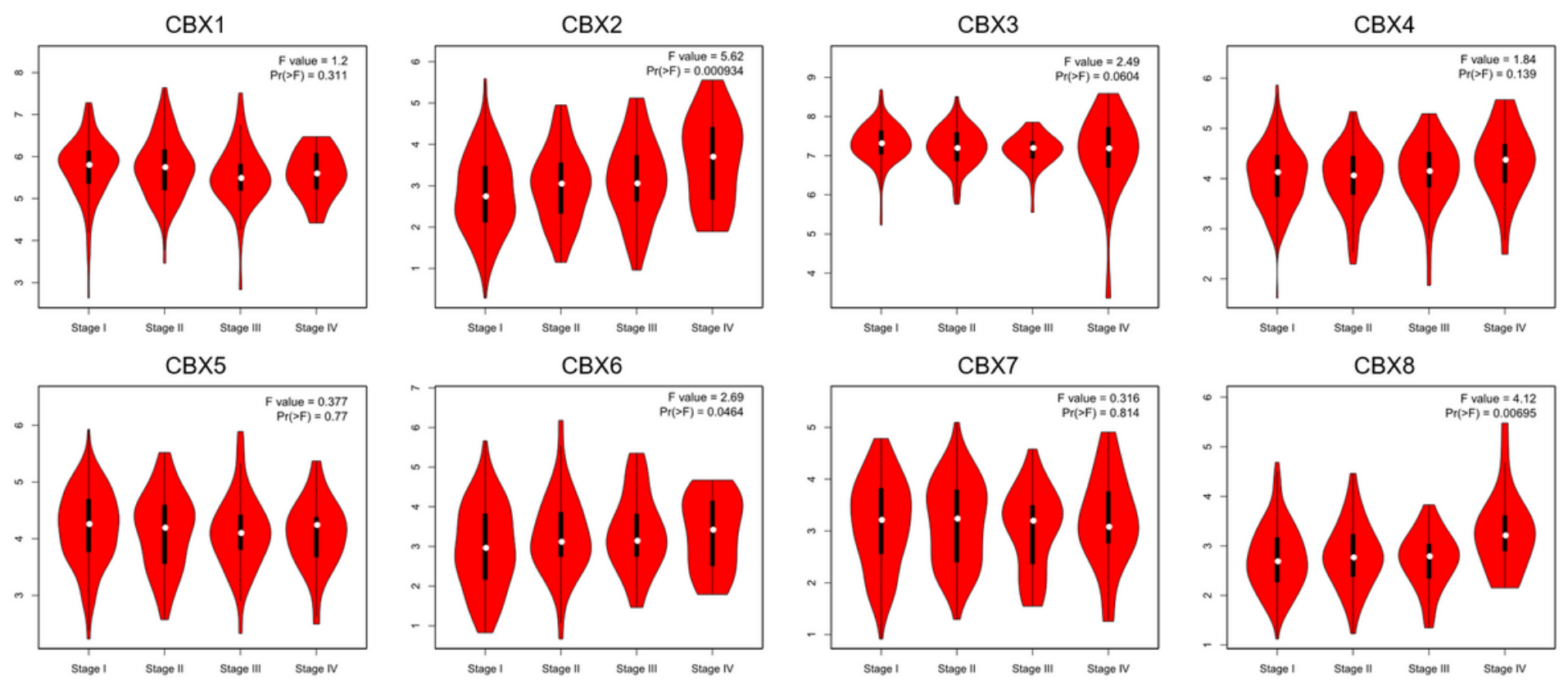

\section{Figure 4}

Relevance between mRNA expression of distinct CBX family members and cancer stages of CESC patients presented by the violin plot. The mRNA expression levels of CBX2/6/8 was associated with the pathological stage in CESC with a p-value $<0.05$.

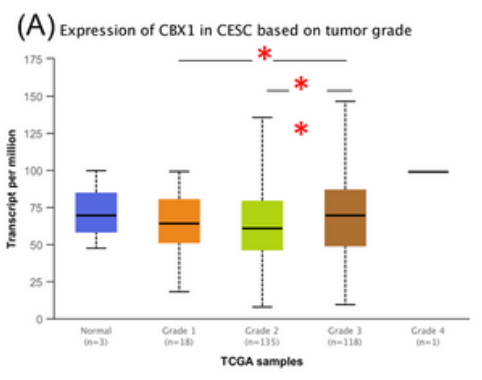

(E) Expression of CBX5 in CESC based on tumor grade

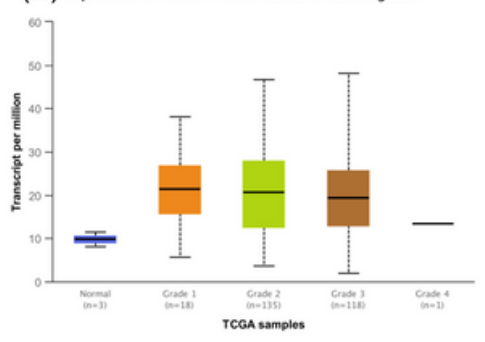

(B) Expression of CBX2 in CESC based on tumor grade

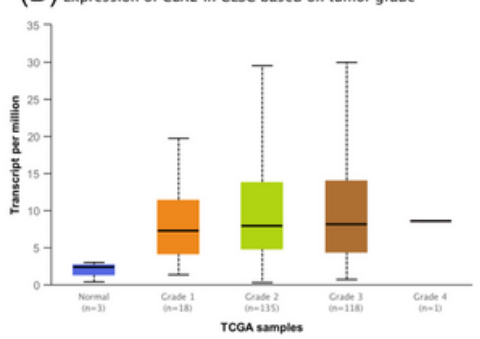

(F) Expression of CBX6 in CESC based on tumor grade

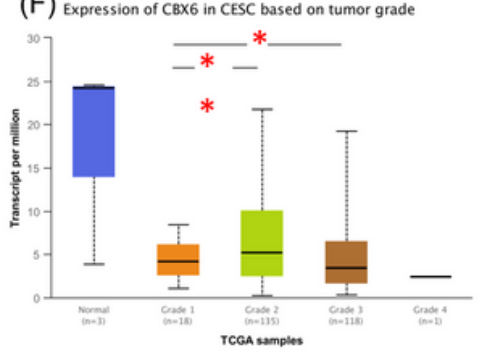

(C) Expression of CBX3 in CESC based on tumor grade

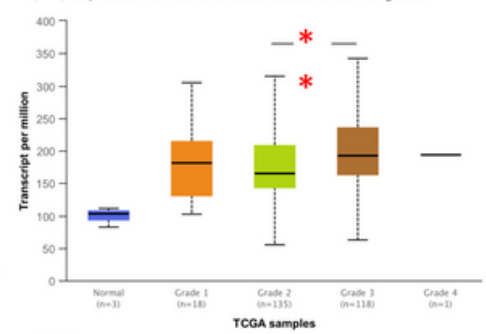

(G) Expression of CBX7 in CESC based on tumor grade

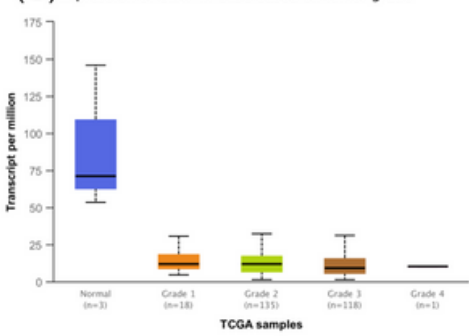

(D) Expression of CBX4 in CESC based on tumor grade

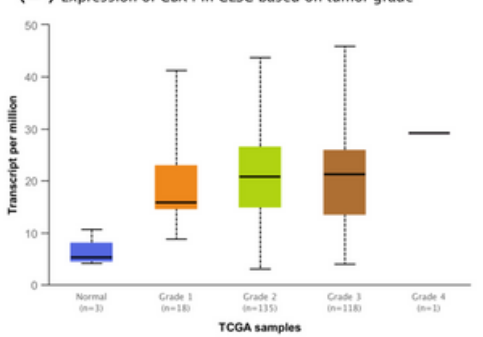

$(\mathrm{H})$ Expression of CBX8 in CESC based on tumor grade

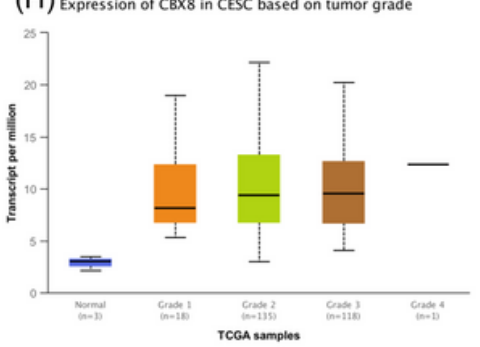

Figure 5 
Relationship of the mRNA expression level of distinct CBX family members with tumor grades of CESC patients. The mRNA expression of $\mathrm{CBX} 1 / 3$ was related to tumor grade, and when the tumor grade increased, the mRNA expression level of $\mathrm{CBX} 1 / 3$ tended to be higher, while that of $\mathrm{CBX} 6$ was the opposite. ${ }^{*} \mathrm{p}<0.05,{ }^{* *} \mathrm{p}<0.01$.
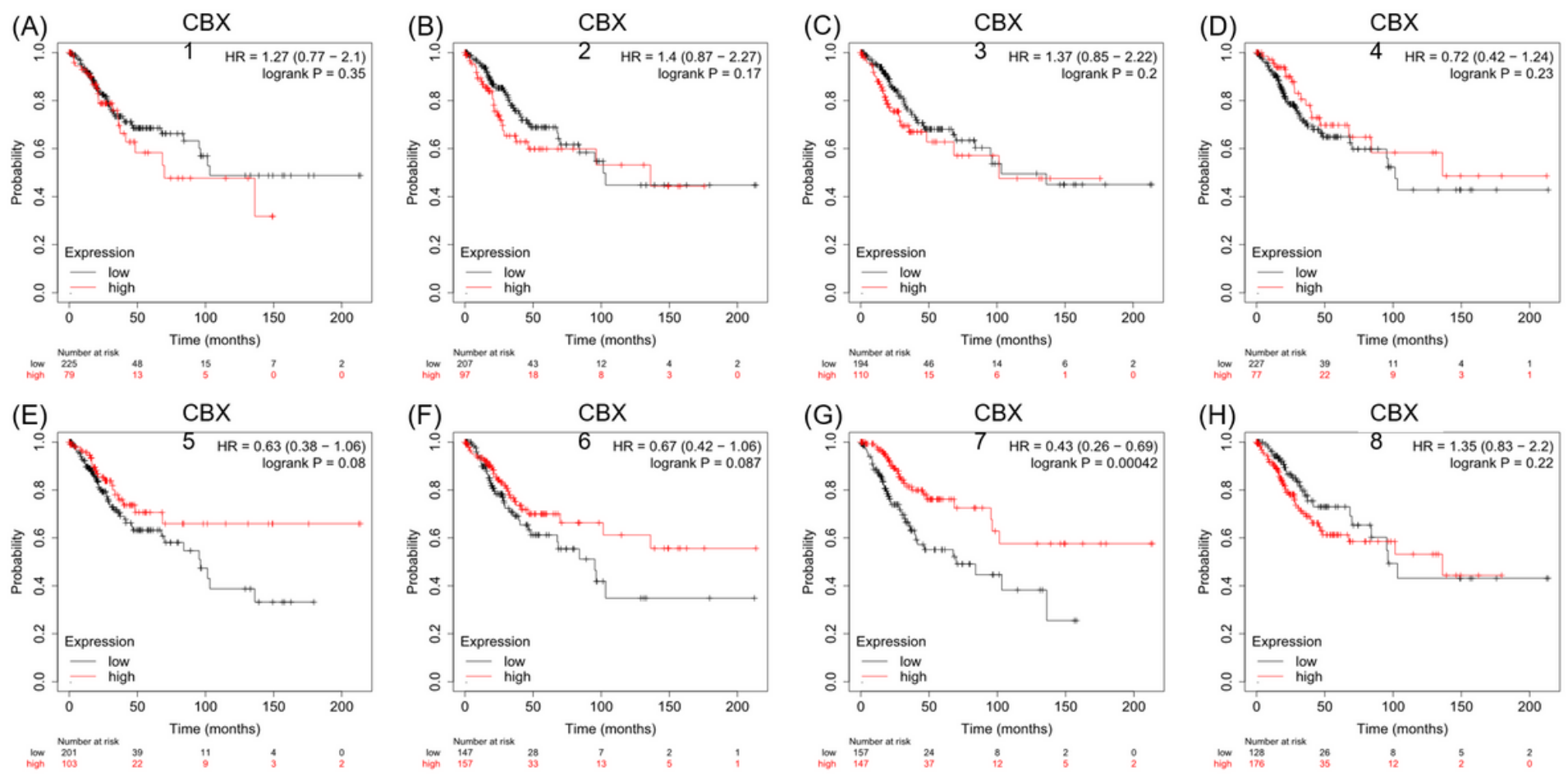

\section{Figure 6}

Prognostic value of the mRNA expression level of CBXs in patients with CESC (Kaplan-Meier plotter). CBX1/2/3/4/5/6/8 mRNA expression showed no correlation with prognosis in CESC patients (A-G), while higher mRNA expression of CBX7 was related to a favorable OS in CESC patients $(H)$. 


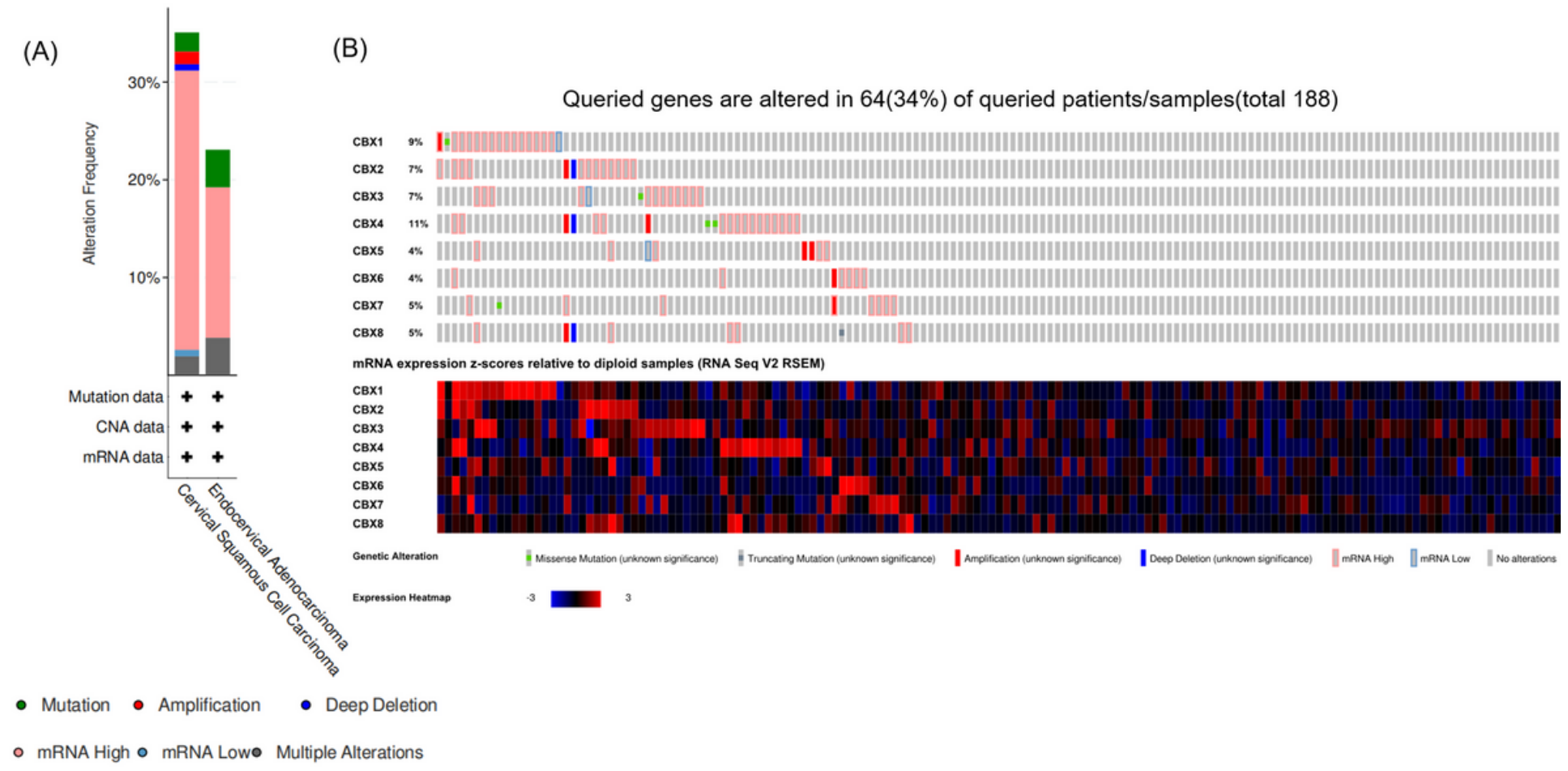

\section{Figure 7}

CBXs gene expression and mutation analysis in CESC (cBioPortal). A high mutation rate (34\%) of CBXs was observed in CESC patients. CBX4, CBX1, CBX2, and CBX3 ranked as the four genes with the most genetic alterations, and their mutation rates were $11 \%, 9 \%, 7 \%$, and $7 \%$, respectively.

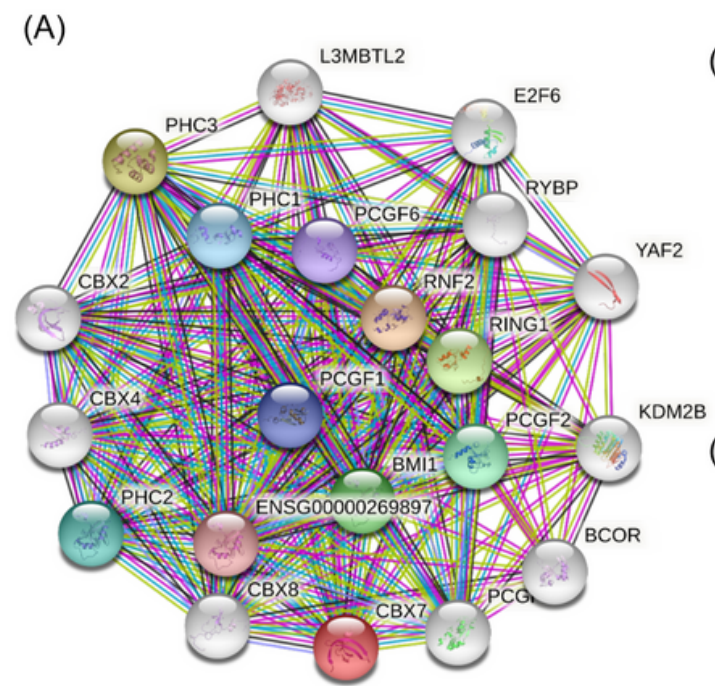

(B)

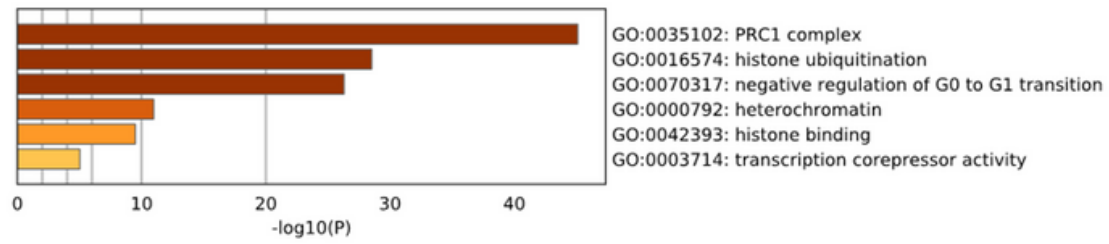

(C)

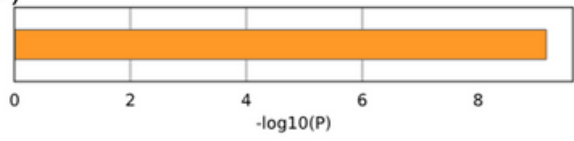

004550: Signaling pathways regulating pluripotency of stem cells

\section{Figure 8}

Predicted functions and pathways of the mutations in CBXs and their frequently altered neighboring genes in CESC patients (A). GO functional enrichment analysis predicted three main functions of CBX mutations and their altered neighboring genes (B). KEGG pathway analysis of CBXs and their altered neighboring genes $(\mathrm{C})$. 